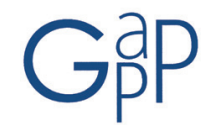

GESTIÓN Y ANÁLISIS DE POLÍTICAS PÚBLICAS, Nueva Época, n 10 julio-diciembre 2013 ISSN: 1989-8991

DOI: http://dx.doi.org/10.24965/gapp.voi10.10109

\title{
Efectos económicos y ambientales del impuesto especial sobre determinados medios de transporte
}

\author{
Jaume Freire-González \\ ENT Medio Ambiente y Gestión \\ jfreire.ent@gmail.com \\ Ignasi Puig-Ventosa \\ ENT Medio Ambiente y Gestión \\ ipuig@ent.cat
}

Recibido: 14 de octubre de 2013

Aceptado: 2 de diciembre de 2013

\begin{abstract}
Resumen
El principal objetivo de la reforma del Impuesto Especial sobre Determinados Medios de Transporte (IEDMT), que entró en vigor en enero de 2008, fue incentivar un progresivo cambio en el parque automovilístico español hacia vehículos menos contaminantes. La presente investigación realiza una estimación, a partir de técnicas econométricas, de los efectos económicos y ambientales de la nueva configuración del IEDMT. La reforma del impuesto ha tenido un efecto positivo y significativo sobre la reducción de las emisiones de $\mathrm{CO}_{2}$ de los nuevos vehículos matriculados.
\end{abstract}

Palabras clave

Impuesto Especial sobre Determinados Medios de Transporte, impuesto de matriculación, fiscalidad ambiental, política de cambio climático

\section{Economic and environmental effects of the vehicle registration tax in Spain}

\section{Abstract}

The main objective of the reform of the Vehicle Registration Tax (IEDMT) in Spain, which came into force in January 2008 , was to encourage a gradual change in the Spanish fleet towards less polluting vehicles. This research estimates the economic and environmental effects of the new configuration of IEDMT using econometric techniques. The tax reform had a positive and significant effect on the reduction of $\mathrm{CO}_{2}$ emissions of new registered vehicles.

\section{Keywords}

Vehicle registration tax, environmental taxation, climate change policy 


\section{INTRODUCCIÓN}

La creciente preocupación por las presiones sobre el medio ambiente ha llevado a los poderes públicos a diseñar instrumentos de política ambiental que reduzcan y limiten los efectos negativos de las emisiones de contaminantes, la generación de residuos, el consumo de recursos naturales y otros impactos perjudiciales para el medio ambiente. Estos instrumentos pueden englobarse en tres grandes grupos: los normativos, básicamente instrumentalizados a través de prohibiciones o limitaciones al uso de determinados recursos, a la emisión de determinados contaminantes, etc.; los voluntarios, destinados a modificar comportamientos, a través de campañas de concienciación, formación, acuerdos voluntarios, etc.; y los instrumentos económicos, consistentes en la utilización de mecanismos de mercado (por ejemplo: impuestos, permisos de contaminación negociables, subvenciones, etc.) con el fin de provocar modificaciones en los costes relativos de determinados bienes y servicios. Estos últimos tendrían el fin de incluir los costes sociales y ambientales en los precios de mercado y, por consiguiente, incentivar o desincentivar su consumo.

Uno de los fallos del mercado considerados en la literatura económica es el de las externalidades. Este se produce cuando las actividades de consumo o producción de un agente provocan costes o beneficios que recaen sobre terceros. Las externalidades afectan a la eficiencia asignativa, ya que los precios no reflejan correctamente todos los costes o beneficios sociales de una determinada actuación de consumo o producción. Las problemáticas ambientales pueden frecuentemente entenderse como externalidades negativas, en la medida que se provocan efectos negativos sobre el bienestar de terceros. A nivel teórico, un impuesto que incrementara el precio lo suficiente, igualaría el coste marginal privado al coste marginal social, reduciendo el consumo de un determinado bien contaminante, y por lo tanto compensando la externalidad y el efecto negativo sobre el bienestar. El principal objetivo de la fiscalidad ambiental es modificar determinados precios de mercado para que estos ofrezcan señales más adecuadas a los agentes y, así, mejorar la asignación de recursos en la economía, reduciendo con ello las presiones medioambientales. En este caso, la recaudación sería un efecto secundario.

En lo que se refiere a la fiscalidad ambiental relacionada con el transporte, en España existe desde 1993 el Impuesto Especial sobre Determinados Medios de Transporte (IEDMT), pero no fue hasta 2008 que este impuesto fue reformado para adoptar propiamente la configuración de impuesto ambiental. Es decir, con el objetivo específico de reducir las emisiones de contaminantes a la atmosfera.

El objetivo del presente artículo es contrastar empíricamente si la citada modificación del IEDMT ha tenido efectos sobre el tipo de vehículos adquiridos, favoreciendo a los vehículos menos contaminantes, así como cuantificar los efectos que ha tenido dicha modificación sobre las emisiones medias de contaminantes de los nuevos vehículos vendidos, estimando los efectos que ha tenido sobre la configuración del parque de vehículos.

Para ello, el apartado 2 realiza un análisis de la situación de la fiscalidad de la matriculación de los vehículos en España, el apartado 3 realiza una descripción de los datos utilizados, el apartado 4 se centra en la especificación y estimación de los modelos econométricos que permiten cuantificar los efectos del IEDMT sobre la venta de vehículos y el apartado 5 contiene las principales conclusiones.

\section{EL IMPUESTO ESPECIAL SOBRE DETERMINADOS MEDIOS DE TRANSPORTE (IEDMT)}

Aparte de los impuestos generales que pueden afectar al automóvil (como el IVA o el impuesto de transmisiones patrimoniales), la fiscalidad de los automóviles se compone básicamente de dos figuras impositivas: el IEDMT (más conocido como impuesto de matriculación) y el impuesto sobre vehículos de tracción mecánica (IVTM) (más conocido como impuesto de circulación). Por otro lado, también es de suma relevancia la fiscalidad sobre los hidrocarburos, puesto que incide directamente sobre el uso de los automóviles, concretamente el Impuesto sobre hidrocarburos y el Impuesto sobre ventas minoristas de determinados hidrocarburos. De todos ellos, el presente artículo se centra en el IEDMT.

El Impuesto Especial sobre Determinados Medios de Transporte (IEDMT) fue creado por la Ley 38/1992, de 28 de diciembre, de Impuestos Especiales. El impuesto grava la primera matriculación en España de medios de transporte, ya sean terrestres, marítimos o aeronáuticos. El IEDMT es un impuesto que se creó para mantener la recaudación asociada a los vehículos, ya que en ese año se suprimió el tipo incrementado del IVA del 28\%, que gravaba determinados medios de transporte. En 2002 se cedió la recaudación del impuesto a las Comunidades Autónomas.

La base imponible del impuesto es el importe de adquisición del medio de transporte, sobre la que se aplica el tipo impositivo correspondiente. Hasta 2007, el tipo era de un $7 \%$ para los vehículos automóviles de turismo de cilindrada inferior a $1.600 \mathrm{~cm}^{3}$ si estaban equipados con un motor de gasolina $02.000 \mathrm{~cm}^{3}$ si estaban equipados con motor diesel, y de un 12\% para el resto de medios de transporte. En Canarias los tipos eran del $6 \%$ y del 11\%, respectivamente, mientras que en Ceuta y Melilla aplicaban unos tipos impositivos nulos. Cabe destacar, como indica 
Cornejo Pérez (2008), que la Ley 34/2007, de 15 de noviembre, de calidad del aire y protección de la atmósfera y su modificación mediante la Ley 51/2007, de 26 de diciembre, de Presupuestos Generales del Estado para el año 2008, en su Disposición adicional octava, disponía una reestructuración del IEDMT sobre la base de los siguientes principios:

- Los tipos de gravamen se establecerían en función de las emisiones de $\mathrm{CO}_{2}$ por kilómetro recorrido.

- $\quad$ El tipo para los vehículos de menos emisores sería cero.

- $\quad$ El tipo para los vehículos de más emisores sería superior al tipo más alto vigente el 30 de junio de 2007.

- Las motos acuáticas y los “quads” tendrían el tipo más alto de los considerados.

A estos principios debía añadirse el principio de neutralidad impositiva, de forma que la recaudación obtenida por el impuesto una vez modificado fuera la misma que antes de dicha reestructuración. Para determinar los tipos impositivos, la Ley 38/1992, de 28 de diciembre, de Impuestos Especiales, en su actual redacción, determina nueve epígrafes diferentes donde se clasifican los vehículos, de menos a más contaminantes. Los tipos son los que, conforme a lo previsto en el artículo 43 de la Ley 21/2001, de 27 de diciembre, por la que se regulan las medidas fiscales y administrativas del nuevo sistema de financiación de las Comunidades Autónomas de régimen común y Ciudades con Estatuto de Autonomía, hayan sido aprobados por la Comunidad Autónoma. A partir de estas categorías se previeron cinco tipos impositivos diferentes, crecientes con las emisiones.

En caso de que la Comunidad Autónoma no haya aprobado sus propios tipos, los tipos impositivos de los distintos epígrafes son los mostrados en la Tabla 1.

TABLA 1

TIPOS IMPOSITIVOS, APLICADOS A LOS VEHÍ́CULOS EN ESPAÑA EN FUNCIÓN DE LAS EMISIONES DE CO,

SI LA COMUNIDAD AUTÓNOMA NO HUBIESE APROBADO TIPOS PROPIOS

\begin{tabular}{|c|c|c|}
\hline \multirow{2}{*}{ Epígrafe } & \multicolumn{2}{|c|}{ Tipo impositivo } \\
\cline { 2 - 3 } & $\begin{array}{c}\text { Península e } \\
\text { Islas Baleares }\end{array}$ & Canarias \\
\hline $1^{\circ}$ y $6^{\circ}$ & $0 \%$ & $0 \%$ \\
\hline $2^{\circ} \mathrm{y}^{\circ}$ & $4,75 \%$ & $3,75 \%$ \\
\hline $3^{\circ}$ y $8^{\circ}$ & $9,75 \%$ & $8,75 \%$ \\
\hline $4^{\circ}$ y $9^{\circ}$ & $14,75 \%$ & $13,75 \%$ \\
\hline $5^{\circ}$ & $12 \%$ & $11 \%$ \\
\hline
\end{tabular}

Notas: En Ceuta y Melilla el tipo será del 0\% para todos los epígrafes.

FUeNTE: Ley 38/1992, de 28 de diciembre, de Impuestos Especiales.

\section{MODELOS ECONOMÉTRICOS Y ESTIMACIONES}

En este apartado se estiman los efectos que ha tenido la modificación del IEDMT sobre diversas variables. En primer término se realiza una breve revisión de la literatura sobre modelos de demanda de vehículos y se sientan las bases metodológicas de un modelo que servirá para llevar a cabo las estimaciones.

Dado que el objeto del estudio es la identificación de los efectos que tuvo la reforma del IEDMT, se han utilizado los datos del periodo 2008-2010 para llevar a cabo las estimaciones, ya que fue en 2008 cuando entró en vigor. A pesar de que una serie más larga permitiría observar un cambio estructural en los efectos del impuesto a partir de 2008, la limitación en ligar las diferentes variables para los años anteriores a 2008 reducía considerablemente la muestra. Después de ligar los datos provenientes de diferentes fuentes quedó, finalmente, una muestra de 59 modelos de vehículos para los años 2008, 2009 y 2010, lo que ha proporcionado una muestra de 177 observaciones, dado que para la variable "emisiones de $\mathrm{CO}_{2}$ solamente se disponía información para algunos modelos (ver anexo de datos). La reducción de la muestra final respecto la inicial se debe a diferencias en la agregación de datos de las diferentes fuentes (específicamente al referirse a marca, modelo y submodelo) y a la no homogeneización de los mismos, apareciendo diferentes modelos en las variables, con diferentes características, etc. 


\section{Revisión de literatura y metodología}

Desde mediados del siglo XX hay cierta literatura empírica que ha desarrollado modelos de demanda para los vehículos de manera consistente. Chow (1957) propuso una ecuación de demanda lineal para la demanda de vehículos, como función de la renta, los precios y las ventas de periodos anteriores. Wetzel y Hoffer (1982) también desarrollaron modelos de demanda y utilizaron análisis de regresión para determinar las preferencias de los consumidores para determinados bienes, incluyendo vehículos. Irvine (1983) calculó elasticidades cruzadas entre modelos específicos de vehículos con diferentes atributos.

Berry et al. (1995) generalizaron los modelos de demanda de vehículos de los estudios previos. Expresaron la función de utilidad del consumidor indirecta para un automóvil como función de las características de los individuos y del producto. Utilizaron un modelo de elección discreta flexible. Para ellos, en un mercado en equilibrio, la utilidad del consumidor i para un vehículo j se puede expresar como:

$$
U_{i j}=f\left(p_{j}, x_{j}, \mu_{j}, y_{i} ; \lambda\right)
$$

Donde $p_{j}$ es el precio del vehículo $j, x_{j}$ son las características observadas del vehículo, $\mu_{j}$ son las características no observadas del vehículo, $y_{i}$ son las características socioeconómicas de los individuos y $\lambda$ es el vector de parámetros a estimar.

El consumidor i adquirirá el vehículo j solo si:

$$
\begin{aligned}
& U_{i j}=f\left(p_{j}, x_{j}, \mu_{j}, y_{i} ; \lambda\right) \geq U_{i r}\left(p_{r}, x_{r}, \mu_{r}, y_{r} ; \lambda\right) \\
& \text { Para } \mathrm{r}=1,2, \mathrm{~K}, \mathrm{n} ; \mathrm{r} \neq \mathrm{j}
\end{aligned}
$$

Dados estos elementos, se puede considerar la demanda agregada para un vehículo en concreto como la suma de las demandas individuales. Por otra parte, la dificultad en la obtención de los datos necesarios, espe-

cialmente para las variables de características: $x_{j}$ y $\mu_{j}$, en una especificación como la mostrada, dificultan las estimaciones. Se propone resolverlo es a partir de datos sobre los modelos individuales, los cuales ya tienen características específicas. A partir de estas consideraciones, de modo muy simple, se puede establecer una función de demanda de vehículos del siguiente tipo:

$$
C_{i t}=f\left(p_{i t}, p_{j t}, i_{i t}, y_{i t}, z_{i t}\right)
$$

Donde $C_{i t}$ es el número de vehículos de la marca y modelo i en el periodo $t, p_{i t}$ es el precio de los vehículos de la marca y modelo $i$ en el periodo $t, p_{i t}$ es el precio de otros bienes substitutivos $j$ en el periodo $t$, $i_{i t}$ es el tipo de interés medio de mercado para la financiación de vehículos de la marca y modelo i en el periodo $t, y_{i t}$ es la renta disponible de los hogares en el periodo $t$ y $z_{i t}$ son otros factores relevantes y específicos para la demanda de vehículos de la marca y modelo i en el periodo $t$.

En esta modelización cabe la consideración adicional de que el precio de adquisición esté formado por dos componentes: el precio básico del vehículo $(p b)$ y el impuesto correspondiente (Q), que a su vez depende del tipo de gravamen:

$$
p_{i t}=f\left(p b_{i t}, Q_{i t}\right)
$$

Para el caso de España, los impuestos que forman parte del precio de adquisición del vehículo son el IVA y el IEDMT, y se obtiene de multiplicar la base imponible por el tipo correspondiente aplicable a cada vehículo:

$$
Q_{i t}=p b_{i t} t_{i t}
$$


Donde $t_{i t}$ es el tipo impositivo correspondiente a la marca y modelo $i$ en el periodo t. A pesar que hay otros aspectos que afectarían la demanda de vehículos, como podrían ser algunos costes de mantenimiento, otras tasas e impuestos, costes del combustible, ${ }^{1}$ etc., se supondrá que estos no tienen una influencia significativa o bien que no varían demasiado entre marcas y modelos de vehículos para los años en consideración. Esta hipótesis es razonable en los modelos planteados dado que estas variables no presentan variaciones significativas entre modelos de vehículos.

Finalmente, cabe destacar algunos trabajos más recientes llevados a cabo con el fin de estimar funciones de demanda de vehículos para observar los efectos de cambios en los precios del combustible u otros (Knittel, 2011; Langer y Miller, 2013), o determinar los efectos de impuestos ambientales sobre la venta de automóviles (Craft y Schmidt, 2005; Beck et al., 2013).

\section{Modelos econométricos y estimaciones}

A partir de las bases teóricas desarrolladas en el apartado anterior, se han especificado y estimado algunos modelos econométricos. Para realizar las estimaciones de los modelos de demanda de vehículos, que determinarían los efectos del IEDMT, se ha realizado en primer término, la siguiente especificación funcional general:

$$
m_{i t}=\alpha_{i t}+\beta_{1} p_{i t}+\beta_{2} t_{i t}+\beta_{3} r_{i t}+\beta_{4} i_{i t}+u_{i t}
$$

Donde $m_{i t}$ son las matriculaciones del modelo $i$ en el año $t, p_{i t}$ es el precio del modelo i en el año $t$, $t_{i t}$ es el impuesto de matriculación del modelo $i$ en el año $t, r_{i t}$ es la renta media del comprador del modelo $i$ en el año $t$, $i_{t}$ es el tipo de interés medio para la financiación del modelo i en el año $t, m_{i t-1}$ son las matriculaciones del modelo $i$ en el periodo t-1 y $u_{i t}$ es el término de perturbación de las estimaciones. Este modelo incluye las principales variables contempladas en los modelos teóricos desarrollados en el apartado anterior.

Este modelo también puede especificarse utilizando las emisiones de $\mathrm{CO}_{2}$ como variable exógena, en vez de la variable correspondiente al impuesto de matriculación. Esta estrategia permite observar los efectos de una variación de los niveles de contaminación sobre la matriculación de vehículos (dado que, a pesar de que los tipos no son lineales con las emisiones, si existe correlación entre ellas). Este sería el modelo general de demanda especificado en primer término, dadas las bases teóricas y la literatura empírica sobre la cuestión, analizadas en el apartado anterior. Se esperaría que los signos de las variables $p, t\left(\mathrm{o} \mathrm{CO}_{2}\right.$, en su caso) e i fueran negativos, es decir, que un incremento de estas redujera las matriculaciones de vehículos, mientras que $r$ tuviera signo positivo.

Se han realizado estimaciones con el panel de 177 observaciones. Se ha descartado la variable correspondiente al tipo de interés por no resultar significativa en ningún análisis. Esto es debido a que no se disponía de datos individuales de esta variable para cada marca de vehículo, sino de datos agregados para toda España.

Finalmente, se utilizaron las especificaciones funcionales que se muestran a continuación para llevar a cabo las estimaciones.

\section{Efectos globales del impuesto de matriculación sobre el número de matriculaciones}

La primera especificación evalúa los efectos globales del impuesto de matriculación sobre el número de matriculaciones de vehículos en el periodo posterior a la entrada en vigor de la modificación del impuesto de matriculación (2008-2010). Se ha especificado el siguiente modelo:

$$
\ln \left(m_{i t}\right)=\alpha_{i}+\beta_{1} \ln \left(p_{i t}\right)+\beta_{2} t_{i t}+\beta_{3} \ln \left(r_{i t}\right)+u_{i t}
$$

Se ha especificado una forma funcional logarítmica para dotar de mayor flexibilidad a los parámetros, así como para obtener los coeficientes estimados en términos de elasticidades, es decir de variaciones porcentuales de las variables exógenas sobre la endógena. El hecho de que, para algunos casos, el impuesto de matriculación tome un valor de 0 , no ha permitido establecer esta variable en logaritmos. Para aprovechar la mayor cantidad de información que proporcionan los datos de panel, se ha especificado un modelo de efectos fijos el cual se ha estimado por el método de los Mínimos Cuadrados Generalizados con la utilización de ponderaciones cross-section (cross-section weights). A continuación, la Tabla 2 muestra los resultados de las estimaciones llevadas a cabo.

1 Esta variable podría empezar a tomar relevancia, y por lo tanto debería introducirse en los análisis, especialmente a partir de la introducción de los vehículos que utilizan fuentes de energía alternativas como los híbridos y los eléctricos. Para un período corto de análisis (2008-2010) se realiza la hipótesis de que no afecta significativamente. 
TABLA 2

ESTIMACIÓN DEL MODELO DE DEMANDA DE VEHÍCULOSCON LA UTILIZACIÓN DEL TIPO IMPOSITIVO DEL IEDMT

MODELO DE EFECTOS FIJOS (MCG CROSS-SECTION WEIGHTS)

\begin{tabular}{lr|}
\hline \multicolumn{1}{|c}{ Variable independiente } & Ln (matriculaciones) \\
\hline Constante & $-192,023 * *(18,124)$ \\
Ln(precio) & $-1,714 *(1,055)$ \\
Impuesto & $-0,001 *(0,000)$ \\
Ln(renta) & $15,858 *(1,123)$ \\
\hline$R^{2}$ ajustado & 0,93 \\
\hline
\end{tabular}

Nota: Desviaciones estándar en paréntesis. Las estimaciones solo muestran los coeficientes comunes a todos los vehículos

* ** Indica que el coeficiente estimado es significativamente diferente de cero al $90 \%$ y $95 \%$ de nivel de confianza, respectivamente.

Se observa como los signos de los coeficientes son los esperados. Los coeficientes resultan individual y conjuntamente significativos. Por otra parte, las estimaciones presentan un coeficiente de determinación $\left(R^{2}\right)$ cercano muy elevado debido elevado grado de ajuste que representa un modelo de efectos fijos con muchas observaciones trasversales. De la estimación se desprendería que el tipo impositivo del impuesto de matriculación ha tenido un efecto negativo y significativo sobre la venta global de vehículos (a mayor tipo, menos ventas), aunque el parámetro estimado no resulta muy estable, ya que al probar otras formas funcionales no resulta globalmente significativo.

\section{Efectos globales de las emisiones de $\mathrm{CO}_{2}$ de los vehículos sobre el número de matriculaciones}

La segunda especificación realizada evalúa los efectos de las emisiones de $\mathrm{CO}_{2}$ sobre el número de matriculaciones en el periodo posterior a la entrada en vigor la modificación del impuesto de matriculación (2008-2010). El modelo especificado es el siguiente:

$$
\ln \left(m_{i t}\right)=\alpha_{i}+\beta_{1} \ln \left(p_{i t}\right)+\beta_{2} \ln \left(C O 2_{i t}\right)+\beta_{3} \ln \left(r_{i t}\right)+u_{i t}
$$

En este caso, se ha substituido la variable correspondiente al impuesto por la variable correspondiente a las emisiones $\mathrm{CO}_{2}$ (expresadas en gramos por $\mathrm{km}$ ) $\left(\mathrm{CO}_{2}\right.$ it), para captar de manera directa los efectos que han tenido las emisiones de $\mathrm{CO}_{2}$ sobre las matriculaciones de vehículos desde la modificación del impuesto. Del mismo modo que en la estimación anterior, se ha especificado una forma funcional logarítmica para dotar de mayor flexibilidad a los parámetros y obtener los coeficientes estimados en términos de elasticidades. En este caso, la variable $\mathrm{CO}_{2}$ puede especificarse también en términos logarítmicos, ya que presenta valores positivos en toda la muestra, a diferencia de la utilización de la variable $t$. Del mismo modo que el modelo anterior, se ha especificado un modelo de efectos fijos estimado por el método de Mínimos Cuadrados Generalizados con la utilización de ponderaciones cross-section (cross-section weights). La Tabla 3 muestra los resultados de las estimaciones.

TABLA 3

ESTIMACIÓN DEL MODELO DE DEMANDA DE VEHÍCULOS CON LA UTILIZACIÓN DEL LAS EMISIONES DE CO 2 MODELO DE EFECTOS FIJOS (MCG CROSS-SECTION WEIGHTS)

\begin{tabular}{lr}
\hline \multicolumn{1}{|c}{ Variable independiente } & Ln (matriculaciones) \\
\hline Constante & $-202,458 * *(19,038)$ \\
$\operatorname{Ln}($ precio $)$ & $-1,645^{*}(1,420)$ \\
$\operatorname{Ln}\left(\mathrm{CO}_{2}\right)$ & $-0,023^{* *}(0,004)$ \\
$\operatorname{Ln}($ renta) & $16,811 * *(1,266)$ \\
\hline $\mathrm{R}^{2}$ ajustado & 0,93 \\
\hline
\end{tabular}

Nota: Desviaciones estándar en paréntesis. Las estimaciones solo muestran los coeficientes comunes a todos los vehículos

$*$ ** indica que el coeficiente estimado es significativamente diferente de cero al $90 \%$ y $95 \%$ de nivel de confianza, respectivamente.

GESTIÓN Y ANÁLISIS DE POLÍTICAS PÚBLICAS, Nueva Época, nº 10 julio-diciembre 2013 ISSN: 1989-8991 - DOI: 10.24965/gapp.voi10.10109 
Se observa que los signos de los coeficientes también son los esperados. Los coeficientes resultan significativos tanto a nivel individual como en conjunto. Por otra parte, las estimaciones presentan un coeficiente de determinación $\left(R^{2}\right)$ cercano a 1. De la estimación se desprende que desde la entrada en vigor de la modificación del impuesto de matriculación, las emisiones de $\mathrm{CO}_{2}$ tienen un efecto negativo y significativo sobre la venta global de vehículos, concretamente, cada punto porcentual de incremento en las emisiones de $\mathrm{CO}_{2}$ reduciría las matriculaciones de los modelos en un 0,023\% y al revés.

\section{Efectos individuales de las emisiones de $\mathrm{CO}_{2}$ de los vehículos sobre el número de matriculaciones}

Finalmente se ha realizado una tercera especificación, a partir de la cual se pueden determinar los efectos individuales, es decir, para cada modelo de vehículo, de una modificación en sus emisiones medias de $\mathrm{CO}_{2}$ sobre sus ventas. Esto se puede realizar a partir de una especificación similar a la desarrollada en el apartado anterior:

$$
\ln \left(m_{i t}\right)=\beta_{i} \ln \left(C O 2_{i t}\right)+\beta_{1} \ln \left(p_{i t}\right)+\beta_{2} \ln \left(r_{i t}\right)+u_{i t}
$$

En este caso, en vez de especificar un efecto fijo individual, se especificó individualmente el parámetro correspondiente a las emisiones de $\mathrm{CO}_{2}$ para cada modelo de vehículo. De este modo se puede estimar el efecto que tiene una modificación de las emisiones de $\mathrm{CO}_{2}$ de cada modelo de la muestra sobre su número de matriculaciones, desde la entrada en vigor de la modificación del impuesto. Adicionalmente, se ha añadido la variable renta en esta estimación. A pesar de que es una variable agregada, permitiría controlar parte de los efectos de la propia evolución económica y eliminaría parte del sesgo producido por la omisión de una variable relevante en las estimaciones. Este modelo también se ha estimado por el método de Mínimos Cuadrados Generalizados con la utilización de ponderaciones cross-section (cross-section weights). La Tabla 4 muestra los resultados de las estimaciones.

TABLA 4

ESTIMACIÓN DEL MODELO DE DEMANDA DE VEHÍCULOS CON LA UTILIZACIÓN DE LAS EMISIONES DE CO2

EFECTOS CO2 INDIVIDUALES (MCG CROSS-SECTION WEIGHTS)

\begin{tabular}{lr}
\hline \multicolumn{1}{|c}{ Variable independiente } & Ln(matriculaciones) \\
\hline $\operatorname{Ln}($ precio $)$ & $-14,469 * *(1,127)$ \\
$\operatorname{Ln}($ renta) & $12,117^{* *}(0,827)$ \\
\hline $\mathrm{R}^{2}$ ajustado & 0,92 \\
\hline
\end{tabular}

Nota: Desviaciones estándar en paréntesis. Las estimaciones solo muestran los coeficientes comunes a todos los vehículos. Los coeficientes para el $\mathrm{CO}_{2}$ individuales se muestran en la Tabla 5.

** indica que el coeficiente estimado es significativamente diferente de cero al $99 \%$ de nivel de confianza.

TABLA 5

EFECTOS INDIVIDUALES PARA EL COEFICIENTE DE CO2 PARA CADA MOdELO DE VEHículo DE LA MUESTRA

\begin{tabular}{|l|r|r|r|}
\hline \multicolumn{1}{|c|}{ Marca } & \multicolumn{1}{c|}{ Modelo } & \multicolumn{1}{c|}{ Coeficiente } & \multicolumn{1}{c|}{ Error estándar } \\
\hline Alfa Romeo & 147 & $-4,233$ & 0,533 \\
\hline Alfa Romeo & 159 & $-3,885$ & 0,515 \\
\hline Alfa Romeo & Brera & $-4,345$ & 0,507 \\
\hline Alfa Romeo & Gt & $-4,226$ & 0,520 \\
\hline Alfa Romeo & Spider & $-4,363$ & 0,513 \\
\hline Audi & A5 & $-3,950$ & 0,592 \\
\hline Audi & A6 & $-3,630$ & 0,552 \\
\hline B.M.W. & Serie 3 & $-3,469$ & 0,550 \\
\hline B.M.W. & Serie 6 & $-3,811$ & 0,589 \\
\hline Chevrolet & Lacetti & $-4,219$ & 0,543 \\
\hline Chevrolet & Nubira & $-4,418$ & 0,545 \\
\hline Chevrolet & Epica & $-3,964$ & 0,516 \\
\hline Citroen & C3 & $-4,013$ & 0,584 \\
\hline Citroen & C4 & $-3,629$ & 0,544 \\
\hline
\end{tabular}

GESTIÓN Y ANÁLISIS DE POLÍTICAS PÚBLICAS, Nueva Época, n 10 julio-diciembre 2013 ISSN: 1989-8991 - DOI: 10.24965/gapp.voi10.10109 


\begin{tabular}{|c|c|c|c|}
\hline Citroen & $C_{5}$ & $-3,675$ & 0,524 \\
\hline Citroen & $\mathrm{C} 8$ & $-4,003$ & 0,524 \\
\hline Dacia & Logan & $-4,228$ & 0,583 \\
\hline Ferrari & 430 Spider & $-3,720$ & 0,620 \\
\hline Ferrari & 612 Scaglietti & $-3,595$ & 0,673 \\
\hline Fiat & Panda & $-4,315$ & 0,602 \\
\hline Fiat & Seicento & $-5,928$ & 0,716 \\
\hline Fiat & Grande Punto & $-4,213$ & 0,586 \\
\hline Fiat & Punto & $-5,145$ & 0,643 \\
\hline Fiat & Bravo & $-4,113$ & 0,554 \\
\hline Fiat & Stilo & $-5,133$ & 0,593 \\
\hline Fiat & Croma & $-4,367$ & 0,529 \\
\hline Fiat & Multipla & $-4,588$ & 0,517 \\
\hline Fiat & Ulysse & $-4,422$ & 0,525 \\
\hline Jaguar & X Type & $-4,333$ & 0,559 \\
\hline Jaguar & $x j$ & $-3,899$ & 0,582 \\
\hline Jaguar & $\mathrm{Xk}$ & $-3,795$ & 0,582 \\
\hline Kia & Opirus & $-4,290$ & 0,495 \\
\hline Lancia & Musa & $-4,573$ & 0,567 \\
\hline Lancia & $\mathrm{Y}$ & $-4,741$ & 0,586 \\
\hline Lancia & Phedra & $-4,233$ & 0,528 \\
\hline Nissan & Micra & $-4,129$ & 0,563 \\
\hline Nissan & Note & $-4,287$ & 0,564 \\
\hline Opel & Astra & $-3,653$ & 0,550 \\
\hline Opel & Gt & $-4,261$ & 0,519 \\
\hline Opel & Tigra & $-4,845$ & 0,606 \\
\hline Opel & Meriva & $-4,238$ & 0,562 \\
\hline Renault & Twingo & $-4,639$ & 0,629 \\
\hline Renault & Espace & $-3,936$ & 0,511 \\
\hline Seat & Ibiza & $-3,887$ & 0,592 \\
\hline Seat & Leon & $-3,620$ & 0,539 \\
\hline Seat & Altea & $-3,792$ & 0,545 \\
\hline Seat & Alhambra & $-4,164$ & 0,535 \\
\hline Skoda & Octavia & $-3,992$ & 0,559 \\
\hline Skoda & Superb & $-4,044$ & 0,539 \\
\hline Ssangyong & Rodius & $-3,804$ & 0,506 \\
\hline Subaru & Impreza & $-4,107$ & 0,509 \\
\hline Subaru & Legacy & $-4,306$ & 0,511 \\
\hline Subaru & Outback & $-3,919$ & 0,524 \\
\hline Volkswagen & Fox & $-5,267$ & 0,611 \\
\hline Volkswagen & Eos & $-4,088$ & 0,543 \\
\hline Volkswagen & Golf & $-3,631$ & 0,545 \\
\hline Volkswagen & Phaeton & $-4,008$ & 0,590 \\
\hline Volkswagen & Passat & $-3,525$ & 0,521 \\
\hline Volkswagen & Touran & $-3,888$ & 0,536 \\
\hline
\end{tabular}

En este caso los signos de los coeficientes son los esperados y los coeficientes resultan significativos tanto a nivel individual como conjuntamente, a un nivel de confianza del $99 \%$. La estimación presenta un coeficiente de determinación $\left(\mathrm{R}^{2}\right)$ cercano a 1. Esta estrategia de estimación permite observar, para cada modelo, los efectos 
que han tenido las emisiones de $\mathrm{CO}_{2}$ sobre la venta de vehículos desde la entrada en vigor de la modificación del IEDMT. Todos los coeficientes individuales estimados menos conco resultan negativos, es decir, un incremento de las emisiones de $\mathrm{CO}_{2}$ reduce la venta en los modelos, pero cada modelo tiene una intensidad diferente, a las ventas de algunos les afecta más que a las de otros, y en algunos casos no son significativos. La elasticidad media es de 4,16, es decir que en término medio un incremento de un punto porcentual en las emisiones reduciría un 4,16\% las matriculaciones. Por otra parte, la elasticidad renta obtenida (un valor estimado alrededor de 12) resulta más elevada que otros estudios de elasticidades renta de la demanda de vehículos (como por ejemplo Bordley y McDonald, 1993, donde proporcionan valores alrededor de 3).

Por lo tanto, a pesar de la simplicidad del modelo y de la necesidad de contrastar los resultados obtenidos a medida que se disponga de más datos y de series más largas, se podría afirmar que el actual IEDMT dependiente de las emisiones de $\mathrm{CO}_{2}$ de los vehículos afecta negativamente a la venta de vehículos si estos incrementan sus niveles de emisión de $\mathrm{CO}_{2}$ y positivamente, si los reducen, tal y como cabría esperar.

\section{CONCLUSIONES}

En el presente trabajo se ha realizado un análisis de los efectos que ha podido tener la modificación del Impuesto Especial sobre Determinados Medios de Transporte (IEDMT) en 2008 en España sobre la venta de vehículos, y la nueva configuración del parque automovilístico. Para hacerlo se han desarrollado y estimado modelos de demanda de vehículos, donde la demanda de estos depende del precio, la renta, otras variables y el tributo, o bien de las emisiones de $\mathrm{CO}_{2}$, como variable proxy del efecto de estas sobre las matriculaciones. Las estimaciones realizadas muestran cómo, a partir de 2008, las emisiones de $\mathrm{CO}_{2}$ de los vehículos han resultado determinantes en las matriculaciones de vehículos, afectando negativamente sobre estas, de un modo similar a como lo hace el precio. Esto significaría que la nueva imposición, que depende de las emisiones, habría penalizado a aquellos vehículos más contaminantes y beneficiado a aquellos que lo son menos. De modo similar, aquellos vehículos que reduzcan sus emisiones verán incrementadas sus matriculaciones y a la inversa. Estos efectos incentivarían a medio y largo plazo a los productores a producir coches menos contaminantes, compitiendo en precios pero también en emisiones, con sus competidores.

A pesar de los resultados obtenidos, sería necesario disponer de mayor cantidad de datos y de series más largas para llegar a resultados más concluyentes sobre los efectos de la modificación del impuesto. También resultaría interesante evaluar los efectos del impuesto a nivel macroeconómico y a nivel presupuestario para las arcas del Estado. En este último caso, resultaría de especial interés un análisis coste-beneficio que contemplara los ahorros sociales en términos de ahorro de combustible, dependencia energética, salud y bienestar de la población, en comparación con los costes en términos de recaudación u otros costes sociales de aplicar la modificación del impuesto.

\section{AGRADECIMIENTOS}

Este artículo deriva del estudio "Análisis de los efectos de la reforma del impuesto especial sobre determinados medios de transporte", elaborado en el marco de las ayudas para investigadores externos del Instituto de Estudios Fiscales (IEF).

\section{BIBLIOGRAFÍA}

BECK, M.J., ROSE, J.M. y HenSHER, D.A. (2013). “Environmental Attitudes and Emissions Charging: An example of Policy Implications for Vehicle Choice”. Transportation Research Part a-Policy and Practice, 50: 171-182.

Berry, S., LeVInSOHn, J. y PAKES, A. (1995). “Automobile Prices in Market Equilibrium”. Econometrica, 63(4): 841-890.

BORDLEY, R. y MCDONALD, J. (1993). "Estimating Aggregate Automotive Income Elasticities from the Population Income-Share Elasticity." Journal of Business and Economic Statistics, 11(2): 209-14.

CHOw, G. C. (1957). Demand for Automobiles in the United States: A Study in Consumer Durables. Amsterdam: North-Holland Publishing Company.

CORNEJo Pérez, A., (2008). "La reestructuración del Impuesto Especial sobre Determinados Medios de Transporte en el contexto de la implantación de una fiscalidad verde por el Estado", Estudios financieros, Revista de contabilidad y tributación: Comentarios, casos prácticos, 302: 3-30.

CRAFT, E.D. y SCHMIDT, R. M. (2005). “An Analysis of the Effects of Vehicle Property Taxes on Vehicle Demand”. National Tax Journal, 697-720. 
IRVINE, F. O. Jr. (1983). "Demand Equations for Individual New Car Models Estimated Using Transaction Prices with Implications for Regulatory Issues”. Southern Economic Journal 49(3): 764-782.

KNITTEL, C.R. (2011). "Automobiles on Steroids: Product Attribute Trade-Offs and Technological Progress in the Automobile Sector”. (N. ${ }^{\circ}$ W15162). National Bureau of Economic Research.

LANGer, A. y Miller, N.H. (2013). “Automakers' Short-run Responses to Changing Gasoline Prices" Review of Economics and Statistics, 95(4): 1198-1211.

Wetzel, J. y Hoffer, G. (1982), “Consumer Demand for Automobiles: A Disaggregated Market Approach”. Journal of Consumer Research, 9: 195-199.

\section{ANEXO DE DATOS}

En este apartado se describen los datos de las principales variables relevantes para evaluar las relaciones entre el IEDMT, las ventas de vehículos, sus precios y sus niveles de emisiones. Por una parte se dispone de datos agregados (para el conjunto de España) de la Estadística del Impuesto sobre Matriculación de Vehículos Automóviles de la Agencia Tributaria (AEAT) para los años 2006 a 2009, que proporciona información sobre el total de matriculaciones de vehículos por cilindrada, Comunidad Autónoma, tipo de combustible y emisiones medias de $\mathrm{CO}_{2}$ (en este caso para el período 2008-2010) en gramos por kilómetro.

Por otra parte se dispone de datos desagregados (por modelo de vehículo) de:

Matriculaciones de vehículos por marca y modelo en España para el período 2005-2010, obtenidos de la Asociación Nacional de Importadores de Automóviles, Camiones, Autobuses y Motocicletas (Aniacam).

Precios medios de venta de los vehículos automóviles en España aplicables en la gestión del Impuesto sobre Transmisiones Patrimoniales y Actos Jurídicos Documentados, Impuesto sobre Sucesiones y Donaciones e IEDMT para el período 2005-2010, obtenidos de las órdenes ministeriales en los respectivos Boletines oficiales del Estado2.

Emisiones de CO2 de los vehículos turismo de venta en España por marca y modelo para el periodo 2005-2010, obtenidas del Instituto para la diversificación y ahorro de energía (IDAE, 2005, 2006, 2007, 2008, 2011).

Importe medio del impuesto de matriculación por marcas de vehículos para el periodo 2008-2010, a partir de su cálculo, aplicando las cuotas correspondientes a cada vehículo, en función de su tramo de contaminación, con los datos de emisiones de $\mathrm{CO} 2$.

Renta Nacional Disponible en España para el período 2000-2008. Para esta variable no fue posible la obtención de datos de la renta media disponible de los compradores de los vehículos por marcas, como hubiera sido deseable a efectos de modelización.

Tipos de interés legales aplicados por las entidades de crédito en España para el periodo 2003-2010. Para esta variable tampoco fue posible la obtención de datos del tipo de interés medio aplicado a los compradores de los vehículos por marcas.

2 Orden EHA/4286/2004, de 20 de diciembre, Orden EHA/4046/2005, de 21 de diciembre, Orden EHA/3867/2006, de 13 de diciembre, Orden EHA/3745/2007, de 14 de diciembre, Orden EHA/3697/2008, de 11 de diciembre, Orden EHA/3476/2009, de 17 de diciembre. 